\title{
Electron affinity and excited states of methylglyoxal
}

Yerbolat Dauletyarov, Andrew R. Dixon, Adam A. Wallace, and Andrei Sanov

Citation: The Journal of Chemical Physics 147, 013934 (2017); doi: 10.1063/1.4982948

View online: http://dx.doi.org/10.1063/1.4982948

View Table of Contents: http://aip.scitation.org/toc/jcp/147/1

Published by the American Institute of Physics

\section{Articles you may be interested in}

Perspective: $C_{60}^{+}$and laboratory spectroscopy related to diffuse interstellar bands

The Journal of Chemical Physics 146, 160901 (2017); 10.1063/1.4980119

Influence of long-range Coulomb interaction in velocity map imaging

The Journal of Chemical Physics 147, 013929 (2017); 10.1063/1.4982616

Formaldehyde roaming dynamics: Comparison of quasi-classical trajectory calculations and experiments The Journal of Chemical Physics 147, 013936 (2017); 10.1063/1.4982823

The energy dependence of $\mathrm{CO}(\mathrm{v}, \mathrm{J})$ produced from $\mathrm{H}_{2} \mathrm{CO}$ via the transition state, roaming, and triple fragmentation channels

The Journal of Chemical Physics 147, 013935 (2017); 10.1063/1.4983138

Ion and velocity map imaging for surface dynamics and kinetics

The Journal of Chemical Physics 147, 013939 (2017); 10.1063/1.4983307

Imaging state-to-state reactive scattering in the $\mathrm{Ar}^{+}+\mathrm{H}_{2}$ charge transfer reaction

The Journal of Chemical Physics 147, 013940 (2017); 10.1063/1.4983305

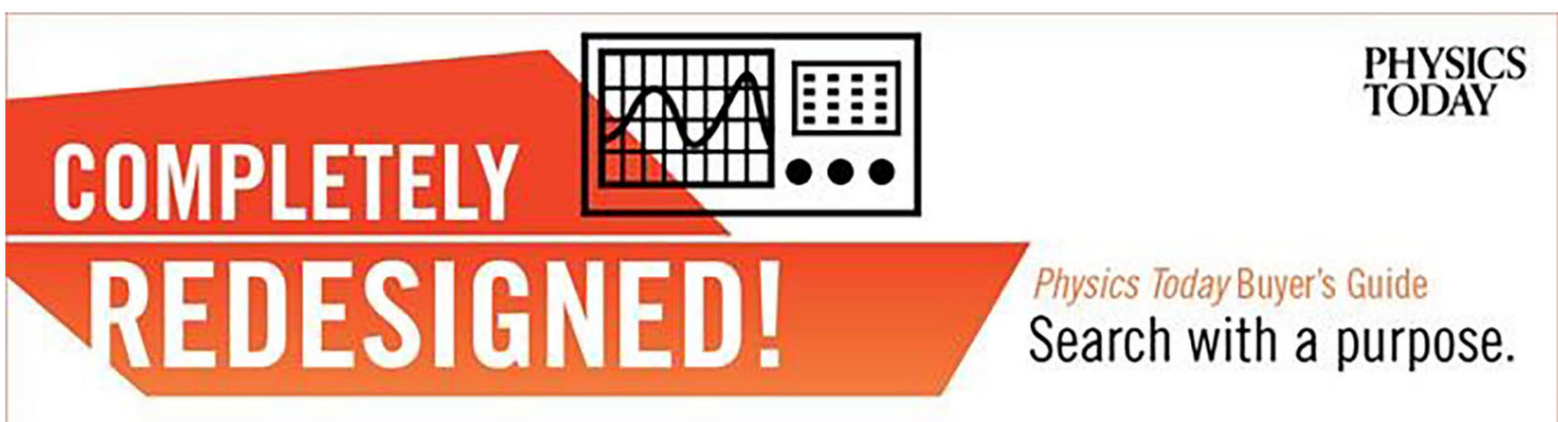




\title{
Electron affinity and excited states of methylglyoxal
}

\author{
Yerbolat Dauletyarov, Andrew R. Dixon, Adam A. Wallace, and Andrei Sanova) \\ Department of Chemistry and Biochemistry, The University of Arizona, Tucson, Arizona 85721, USA
}

(Received 16 March 2017; accepted 22 April 2017; published online 11 May 2017)

\begin{abstract}
Using photoelectron imaging spectroscopy, we characterized the anion of methylglyoxal $\left(X^{2} \mathrm{~A}^{\prime \prime}\right.$ electronic state) and three lowest electronic states of the neutral methylglyoxal molecule: the closed-shell singlet ground state $\left(X^{1} \mathrm{~A}^{\prime}\right)$, the lowest triplet state $\left(a^{3} \mathrm{~A}^{\prime \prime}\right)$, and the open-shell singlet state $\left(A^{1} \mathrm{~A}^{\prime \prime}\right)$. The adiabatic electron affinity (EA) of the ground state, $\mathrm{EA}\left(X^{1} \mathrm{~A}^{\prime}\right)=0.87(1) \mathrm{eV}$, spectroscopically determined for the first time, compares to $1.10(2) \mathrm{eV}$ for unsubstituted glyoxal. The EAs (adiabatic attachment energies) of two excited states of methylglyoxal were also determined: $\operatorname{EA}\left(a^{3} \mathrm{~A}^{\prime \prime}\right)$ $=3.27(2) \mathrm{eV}$ and $\operatorname{EA}\left(A^{1} \mathrm{~A}^{\prime \prime}\right)=3.614(9) \mathrm{eV}$. The photodetachment of the anion to each of these two states produces the neutral species near the respective structural equilibria; hence, the $a^{3} \mathrm{~A}^{\prime \prime} \leftarrow X^{2} \mathrm{~A}^{\prime \prime}$ and $A^{1} \mathrm{~A}^{\prime \prime} \leftarrow X^{2} \mathrm{~A}^{\prime \prime}$ photodetachment transitions are dominated by intense peaks at their respective origins. The lowest-energy photodetachment transition, on the other hand, involves significant geometry relaxation in the $X^{1} \mathrm{~A}^{\prime}$ state, which corresponds to a $60^{\circ}$ internal rotation of the methyl group, compared to the anion structure. Accordingly, the $X^{1} \mathrm{~A}^{\prime} \leftarrow X^{2} \mathrm{~A}^{\prime \prime}$ transition is characterized as a broad, congested band, whose vertical detachment energy, $\mathrm{VDE}=1.20(4) \mathrm{eV}$, significantly exceeds the adiabatic EA. The experimental results are in excellent agreement with the $a b$ initio predictions using several equation-of-motion methodologies, combined with coupled-cluster theory. Published by AIP Publishing. [http://dx.doi.org/10.1063/1.4982948]
\end{abstract}

\section{INTRODUCTION}

Methylglyoxal (MG), $\mathrm{OHCC}\left(\mathrm{CH}_{3}\right) \mathrm{O}$, is one of the small dicarbonyls with important roles in atmospheric chemistry. This work investigates the properties of methylglyoxal (MG) in comparison to its unsubstituted precursor-glyoxal $(\mathrm{G})$, OHCCHO. ${ }^{1}$ Although both G and MG are but minor components of Earth's atmosphere, their significant contributions to the chemistry of volatile organic compounds make them important players in the environment. ${ }^{2-12}$

Despite their relative importance and rather elementary structures, some of the most fundamental properties of the $G$ and MG molecules and their anions have remained unknown or poorly defined. Only recently, the adiabatic electron affinity (EA) of glyoxal was determined directly using anion photoelectron spectroscopy: $\mathrm{EA}(\mathrm{G})=1.10(2) \mathrm{eV} .^{1}$ This result is recommended to replace the only previous experimental (but indirect) estimate ${ }^{12}$ of $0.62(26) \mathrm{eV}$, still cited in most chemistry databases. ${ }^{13}$

For methylglyoxal, no attempts to measure its electron affinity have been reported to date. In 1980, Rawlings and Davidson estimated the EA of MG theoretically. Specifically, they obtained a restricted Hartree-Fock estimate of $\mathrm{EA}(\mathrm{G})$ $\sim 0.9 \mathrm{eV}$ for unsubstituted glyoxal. ${ }^{14}$ Then, based on the comparison to similar methyl-group substitutions, they proposed that the EA(MG) should be $\sim 0.2 \mathrm{eV}$ lower than the above $\mathrm{EA}(\mathrm{G})$ value, thus arriving at an estimate of $\mathrm{EA}(\mathrm{MG})$ $\sim 0.7 \mathrm{eV}$. In the present work, we report a robust experimental determination of the $\mathrm{EA}(\mathrm{MG})=0.87(1) \mathrm{eV}$.

a)Email: sanov@u.arizona.edu
The photochemistry and excited states of MG have received more attention than its anion. Previous laser-induced phosphorescence and fluorescence studies have identified the lowest triplet state of MG adiabatically $2.414 \mathrm{eV}$ above the ground state ${ }^{15}$ and the lowest excited singlet adiabatically $2.743 \mathrm{eV}$ above the ground state. ${ }^{16}$ In the present work, we compare these singlet-triplet and singlet-singlet splittings to the corresponding band intervals in the photoelectron spectrum of the MG anion.

In what follows, anion photoelectron imaging spectroscopy is used to probe the ground (closed-shell singlet $X^{1} \mathrm{~A}^{\prime}$ ) and two excited (lowest triplet $a^{3} \mathrm{~A}^{\prime \prime}$ and open-shell singlet $A^{1} \mathrm{~A}^{\prime \prime}$ ) electronic states of methylglyoxal, accessed from the corresponding radical anion, $\mathrm{MG}^{-}\left(X^{2} \mathrm{~A}^{\prime \prime}\right)$. We report the first experimental determinations of the $\mathrm{EA}$ of $\mathrm{MG}$ in the ground and excited electronic states. The experimental results are supported by $a b$ initio calculations and Franck-Condon simulations and provide insight into the electronic structure of methylglyoxal, in both neutral and anion states.

\section{EXPERIMENTAL AND THEORETICAL METHODS}

The experiments were performed using a custom-built anion photoelectron imaging spectrometer described previously. ${ }^{17,18}$ The methylglyoxal solution $(40 \%$ by weight in water) was first partially dehydrated using $3 \AA$ molecular sieves with a $1: 1$ volume ratio for at least $24 \mathrm{~h}$, until the solution turned yellowish. Unlike our previous experience with glyoxal, ${ }^{1,19,20}$ the methylglyoxal solution did not require any methanol solvent to be extracted from the sieve mixture-it was simply decanted. 
The resulting solution was placed in a sample holder connected to the instrument's ion source chamber. The sample had sufficient vapor pressure at room temperature, so no heating was required. Sample vapor was seeded in an $\mathrm{N}_{2} \mathrm{O}$ carrier gas with a backing pressure of 20 psi. The resulting mixture was expanded into the source chamber through a pulsed supersonic nozzle (General Valve, Inc., Series 9), operated at a $20 \mathrm{~Hz}$ repetition rate matching that of the laser. The supersonic expansion was crossed with a collimated beam of $1 \mathrm{keV}$ electrons from an electron gun. Anions were formed by attachment of slow secondary electrons in the electron-impact ionized plasma. ${ }^{21}$

Anions were extracted into the flight tube of a linear Wiley-McLaren time-of-flight mass-spectrometer, ${ }^{22}$ where they were accelerated to a kinetic energy of $2.5 \mathrm{keV}$, mass selected, and interrogated by $\sim 7$ ns duration laser pulses in the detection region of the instrument. ${ }^{17,18}$ The 812,612, and $306 \mathrm{~nm}$ light was obtained as the fundamental or frequencydoubled output of a Continuum, Inc. ND6000 dye laser pumped by a Surelite II Nd:YAG. The laser pulses were timed to interact only with the anions of a mass-to-charge ratio $\mathrm{m} / \mathrm{z}$ $=72$ (MG). Photoelectrons were velocity-mapped ${ }^{23}$ in the direction perpendicular to the ion and laser beams and projected onto a $40 \mathrm{~mm}$ diameter dual microchannel-plate detector, coupled to a P43 phosphor screen. Photoelectron impact positions were recorded by a thermoelectrically cooled chargecoupled-device camera. Images were typically accumulated for $\sim 10^{6}$ experimental cycles. The complete three-dimensional photoelectron distribution was reconstructed via an inverse Abel transformation ${ }^{24}$ implemented in the BASEX program. ${ }^{25}$ The resulting radial distributions were converted to photoelectron spectra using the known $\mathrm{O}^{-}$photodetachment transitions for calibration. ${ }^{26,27}$

Electronic structure calculations and geometry optimizations were performed using the Q-Chem 4.0 software package. ${ }^{28}$ Details of the calculations are described in Sec. IV. In brief, geometry optimizations were performed using coupled-cluster theory with single and double excitations (CCSD). Vertical energy gaps between different electronic states at the CCSD-optimized geometry of the anion were determined using single-point calculations employing the equation-of-motion (EOM) ionization-potential (IP), electronaffinity (EA), and spin-flip (SF) methodologies, ${ }^{29}$ combined with coupled-cluster theory. In some cases, the EOM calculations were extended to include the non-iterative perturbative diagonal triples (dT) corrections $\mathrm{s}^{30}$ to the CCSD energies. All calculations employed Dunning's augmented correlationconsistent basis set of triple- $\zeta$ quality (aug-cc-pVTZ).

In addition, the CCSD/aug-cc-pVTZ optimized geometries and vibrational frequencies ${ }^{31}$ were used for the normal mode analysis as part of the Franck-Condon (FC) simulation of MG anion photodetachment to the triplet neutral state. The FC simulation was carried out using the PESCAL 2010 software, with the normal modes treated as uncoupled harmonic oscillators with full Duschinsky rotation using the Chen method. $^{32,33}$

\section{EXPERIMENTAL RESULTS}

The anion photoelectron images and corresponding spectra of MG obtained at 812,612, and $306 \mathrm{~nm}$ are shown in Figure 1. The $612 \mathrm{~nm}$ MG spectrum in (a) is presented in comparison with the previously reported $532 \mathrm{~nm}$ anion photoelectron spectrum of unsubstituted glyoxal $(\mathrm{G}) .{ }^{1}$ For the ease of comparison, all spectra are plotted on the electron binding energy (eBE) scale, defined as eBE $=h v-\mathrm{eKE}$, where $h v$ is the photon energy and eKE is electron kinetic energy.

In Figure 1(a), a single photodetachment band labeled A is observed in each of the MG and $G$ spectra. This congested transition is characterized by a predominantly perpendicular photoelectron anisotropy, as seen in the MG images in Figures 1(a) and 1(b) and reported previously for G. ${ }^{1}$ Similar to G, feature A in all MG spectra in Figure 1 is assigned to the transition

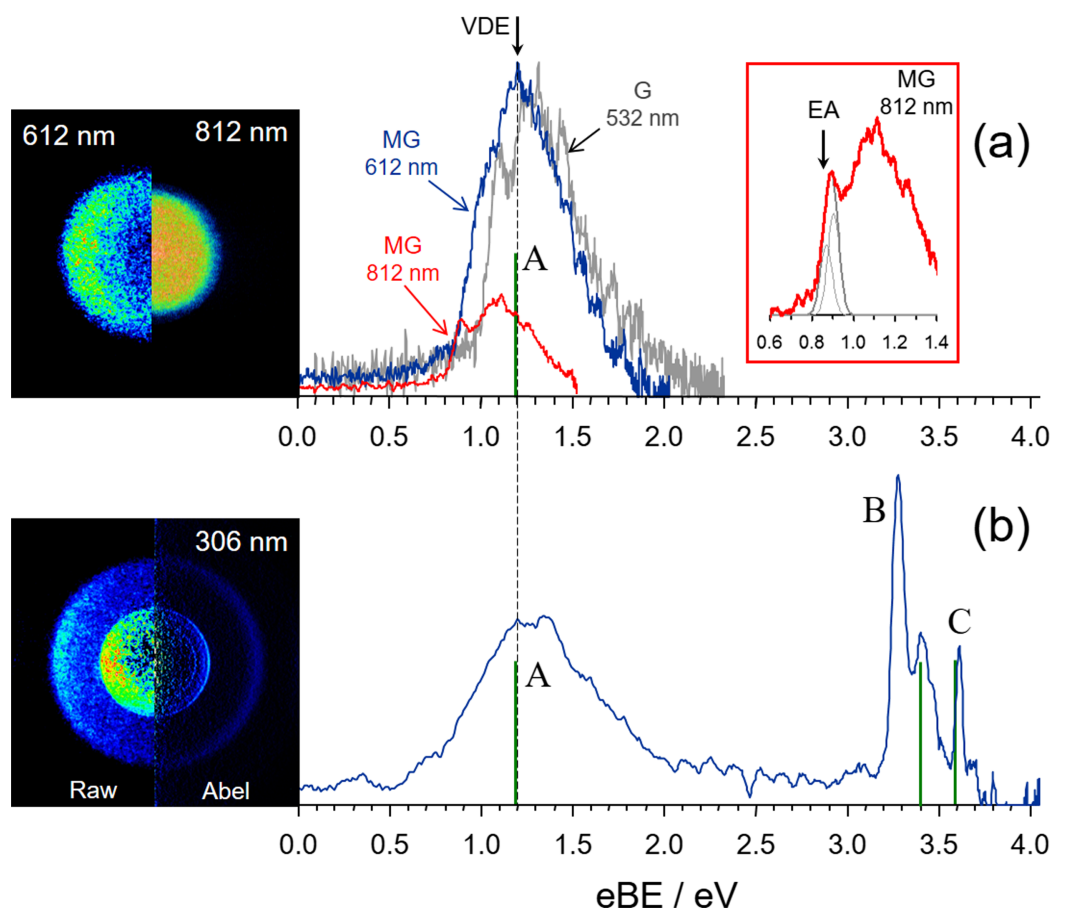

FIG. 1. Anion photoelectron images and spectra of methylglyoxal (MG). (a) Composite of the unprocessed $612 \mathrm{~nm}$ and $812 \mathrm{~nm}$ MG images (left and right halves, respectively) and the corresponding spectra shown in red and blue, as indicated. The $532 \mathrm{~nm}$ anion photoelectron spectrum of unsubstituted glyoxal $(G)$ is also included for comparison (in gray). The inset shows the magnified portion of the $812 \mathrm{~nm}$ MG spectrum, with the vertical arrow indicating the adiabatic electron affinity, EA $=0.87(1) \mathrm{eV}$. (b) The composite $306 \mathrm{~nm}$ anion photoelectron image of $\mathrm{MG}$, consisting of raw (left) and Abel-inverted (right) data, and the corresponding photoelectron spectrum. Band A in all spectra is assigned to the singlet ground state of the neutral molecule. Band B is assigned to the lowest-lying triplet state of MG and band $\mathrm{C}$ to the first excited singlet. The green vertical bars in (a) and (b) indicate the VDEs, calculated at the EOM-IPCCSD/aug-cc-pVTZ level of theory. See Table II and the text for details. 
from the ground state of the anion $\left(X^{2} \mathrm{~A}^{\prime \prime}\right)$ to the ground state of neutral MG $\left(X^{1} \mathrm{~A}^{\prime}\right)$. The $812 \mathrm{~nm} \mathrm{MG}$ spectrum in Figure 1(a) exhibits a clearly identifiable - if only partially resolvedorigin peak of band A, corresponding to the electron affinity (as discussed later in this section).

Band maxima in broad and congested photodetachment transitions generally correspond to vertical detachment energies (VDEs). The width of band $\mathrm{A}$ is due to the significant geometry change between the corresponding anion and neutral equilibria. Based on the band's maximum in the $612 \mathrm{~nm}$ MG spectrum in Figure 1(a), we assign the VDE for the $X^{1} \mathrm{~A}^{\prime}$ $\leftarrow X^{2} \mathrm{~A}^{\prime \prime}$ transition as $1.20(4) \mathrm{eV}$. This value is indicated with the dashed vertical line traversing both Figures 1(a) and 1(b). Note that the shift of the band's maximum in the $812 \mathrm{~nm}$ spectrum relative to $612 \mathrm{~nm}$ is attributed to the centrifugal-barrier suppression of the slow-electron signal in anion photodetachment $^{34}$ in the relative proximity of the photon energy cutoff. The $612 \mathrm{~nm}$ peak position may also be affected by a similar shift, but to a smaller degree (as it is farther from the spectral cutoff) and is hence neglected here.

In addition to band $\mathrm{A}$, two higher-energy transitions are seen in the $306 \mathrm{~nm}$ spectrum in Figure 1(b). In comparison to band $\mathrm{A}$, both of these transitions are characterized by less congested and sharper bands. Band $\mathrm{B}$ is assigned to the lowest triplet state of MG, $a^{3} \mathrm{~A}^{\prime \prime}$. The first and most intense peak of this feature is also its origin, identified as the $0-0$ vibrational transition. This assignment is confirmed by the Franck-Condon analysis in Sec. IV C. The adiabatic detachment energy from the anion to the triplet, i.e., electron affinity of the triplet, is determined from the Franck-Condon modeling of this peak as $\operatorname{EA}\left(a^{3} \mathrm{~A}^{\prime \prime}\right)=3.27(2) \mathrm{eV}$. The even-higher-eBE feature in Figure 1(b), labeled $C$, is assigned to the first excited singlet state of MG, $A^{1} \mathrm{~A}^{\prime \prime}$, with an $\mathrm{EA}\left(A^{1} \mathrm{~A}^{\prime \prime}\right)=3.614(9) \mathrm{eV}$, determined directly from the spectral feature.

We take advantage of the sharp origins of photodetachment bands $\mathrm{B}$ and $\mathrm{C}$, in conjunction with the known singletsinglet and singlet-triplet excitation energies in the neutral, to obtain an accurate and precise determination of the adiabatic EA of the ground state of MG. Specifically, the adiabatic energy gap between the ground and first excited singlet states in MG was previously determined to be 22 125(1.5) $\mathrm{cm}^{-1}(2.743 \mathrm{eV})$, using $A^{1} \mathrm{~A}^{\prime \prime} \leftarrow X^{1} \mathrm{~A}^{\prime}$ fluorescence excitation spectroscopy. ${ }^{16}$ Subtracting this gap from $\operatorname{EA}\left(A^{1} \mathrm{~A}^{\prime \prime}\right)$ $=3.614(9) \mathrm{eV}$, as determined above, we obtain the electron affinity of the ground state, $\operatorname{EA}\left(X^{1} \mathrm{~A}^{\prime}\right)=0.871(9) \mathrm{eV}$. Similarly, the origin of the $a^{3} \mathrm{~A}^{\prime \prime} \leftarrow X^{1} \mathrm{~A}^{\prime}$ transition in MG was observed at $5136 \AA(2.414 \mathrm{eV}) .{ }^{15}$ Subtracting this value of the singlet-triplet splitting from the above EA of the triplet, $\operatorname{EA}\left(a^{3} \mathrm{~A}^{\prime \prime}\right)=3.27(2) \mathrm{eV}$, yields $\mathrm{EA}\left(X^{1} \mathrm{~A}^{\prime}\right)=0.86(2) \mathrm{eV}$.

The two values, $\operatorname{EA}\left(X^{1} \mathrm{~A}^{\prime}\right)=0.871(9) \mathrm{eV}$ and $0.86(2)$ $\mathrm{eV}$, represent direct spectroscopic determinations of adiabatic electron affinity of MG, using different combinations of photoelectron, fluorescence, and phosphorescence spectroscopies. The two results are consistent with each other and with the partially resolved leading peak of band A in the $812 \mathrm{~nm}$ spectrum in Figure 1(a). From these determinations, we assign the electron affinity of MG as $\mathrm{EA}\left(X^{1} \mathrm{~A}^{\prime}\right)=0.87(1) \mathrm{eV}$. The inset in Figure 1(a) shows the result of modeling the band's leading peak with Gaussian functions, assuming this EA value. The agreement between the model and experimental features is excellent.

In comparison to $\mathrm{EA}=0.87(1) \mathrm{eV}$ for $\mathrm{MG}$, the $\mathrm{EA}$ of unsubstituted glyoxal was recently measured using the same experimental methodology to be $1.10(2) \mathrm{eV} .^{1}$ The methyl group has a destabilizing effect on the anion, lowering the EA of MG relative to that of unsubstituted glyoxal. This observation confirms the qualitative prediction of Rawlings and Davidson ${ }^{14}$ and indicates that unsubstituted glyoxal is a more capable electron acceptor in charge-transfer reactions, compared to methylglyoxal.

\section{EXPERIMENT VS. THEORY}

\section{A. Equilibrium structures and adiabatic electron affinities}

The structures of the MG anion $\left(X^{2} \mathrm{~A}^{\prime \prime}\right)$, the neutral closed-shell singlet ground state $\left(X^{1} \mathrm{~A}^{\prime}\right)$, and the lowest triplet state $\left(a^{3} \mathrm{~A}^{\prime \prime}\right)$ were optimized at the CCSD level of theory with the aug-cc-pVTZ basis set using Q-Chem. The resulting structures are shown in Figure 2, while the corresponding geometric parameters are summarized in Table I. As seen in Figure 2, the anion and triplet structures are remarkably similar, while the ground-state singlet differs mainly by an internal rotation of the methyl group of about $60^{\circ}$.

The anion structure maximizes the anionic hydrogen interactions of the $\mathrm{O}$ atom closest to the methyl group with two of its hydrogens $(\mathrm{O} 2-\mathrm{H} 3$ and $\mathrm{O} 2-\mathrm{H} 4$, as labeled in Figure 2), while the third $\mathrm{H}$ interacts with the farther removed $\mathrm{O}$ atom $(\mathrm{O} 1-\mathrm{H} 2)$. This motif is energetically more favorable in the anion, compared to the alternative structure of the ground neutral state. The distinct triplet vs. ground-state singlet structural motifs are
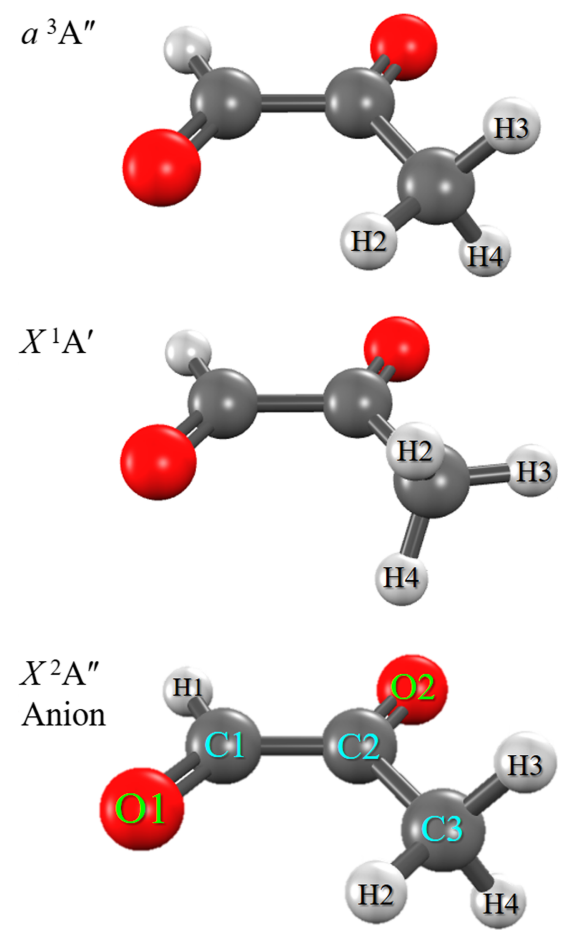

FIG. 2. The optimized minimum-energy structures of the MG anion $\left(X^{2} \mathrm{~A}^{\prime \prime}\right)$ and the neutral in the $X^{1} \mathrm{~A}^{\prime}$ and $a^{3} \mathrm{~A}^{\prime \prime}$ electronic states. The corresponding geometric parameters are reported in Table I. 
TABLE I. Geometric parameters defining the equilibrium geometries of the methylglyoxal anion in the $X^{2} \mathrm{~A}^{\prime \prime}$ state and the neutral methylglyoxal molecule in the $X^{1} \mathrm{~A}^{\prime}$ and $a^{3} \mathrm{~A}^{\prime \prime}$ states, represented pictorially in Figure 2. The structures were optimized using CCSD/aug-cc-pVTZ. The bond lengths are in Angstroms, while the angles are in degrees. Bold emphasis: neutral parameters exhibiting significant deviations from the anion geometry (at least $0.05 \AA$ or $3^{\circ}$ ). Electronic energies relative to the anion structures (i.e., the adiabatic electron affinities) are also given and compared to the experimental values.

\begin{tabular}{lccc}
\hline \hline Parameter & Anion $X^{2} \mathrm{~A}^{\prime \prime}$ & $X^{1} \mathrm{~A}^{\prime}$ & $a^{3} \mathrm{~A}^{\prime \prime}$ \\
\hline $\mathrm{C} 1-\mathrm{C} 2$ & 1.4205 & $\mathbf{1 . 5 2 0 8}$ & $\mathbf{1 . 4 7 6 5}$ \\
$\mathrm{C} 1-\mathrm{O} 1$ & 1.2630 & $\mathbf{1 . 2 0 0 0}$ & 1.2318 \\
$\mathrm{C} 1-\mathrm{H} 1$ & 1.1004 & 1.0961 & 1.0843 \\
$\mathrm{C} 2-\mathrm{O} 2$ & 1.2620 & $\mathbf{1 . 2 0 5 0}$ & 1.2218 \\
$\mathrm{C} 2-\mathrm{C} 3$ & 1.5099 & 1.4924 & 1.5031 \\
$\mathrm{C} 3-\mathrm{H} 2$ & 1.0880 & 1.0883 & 1.0872 \\
$\mathrm{C} 3-\mathrm{H} 3$ & 1.0920 & 1.0841 & 1.0873 \\
O1-C1-C2 & 126.94 & $\mathbf{1 2 2 . 9 0}$ & $\mathbf{1 2 2 . 5 1}$ \\
$\mathrm{H} 1-\mathrm{C} 1-\mathrm{C} 2$ & 113.00 & 113.78 & 114.45 \\
O2-C2-C1 & 123.00 & $\mathbf{1 1 7 . 5 7}$ & 121.33 \\
C3-C2-C1 & 117.79 & 117.24 & 116.31 \\
H2-C3-H3 & 109.60 & 110.52 & 110.62 \\
H3-C3-H4 & 107.33 & 110.52 & 108.69 \\
H2-C3-H4 & 109.60 & 106.82 & 110.62 \\
C2-C3-H2 & 110.89 & 109.56 & 108.32 \\
C2-C3-H3 & 109.67 & 109.81 & 109.28 \\
C1-C2-C3-H2 & 0 & $\mathbf{- 5 8 . 4 4}$ & 0 \\
C1-C2-C3-H3 & -121.19 & $\mathbf{1 8 0}$ & -120.59 \\
\hline CCSD energy/Hartree & -266.830680 & -266.802790 & -266.701914 \\
$\Delta \mathrm{E}-\mathrm{CCSD}$ energy/eV & 0 & 0.759 & 3.504 \\
(relative to anion) & & & $3.27(2)$ \\
Experimental EA/eV & & $0.87(1)$ & \\
\hline \hline
\end{tabular}

consistent with the past results of laser-induced phosphorescence spectroscopy, which show that the triplet transition has a characteristic vibrational progression, indicating an internal rotation of the methyl group in the triplet equilibrium relative to the ground-state singlet. ${ }^{15,16}$

The nominal electron configurations corresponding to these electronic states (as well as the first excited singlet state) are included in Table II. The key molecular orbitals (MOs) defining the differences between the states, namely the $16 a^{\prime}$ and $4 a^{\prime \prime}$ MOs, are presented in Figure 3. The iso-surfaces shown correspond specifically to the $16 a^{\prime}-\alpha($ HOMO-1) and the $4 a^{\prime \prime}-\alpha$ HOMO of the CCSD/aug-cc-pVTZ anion reference at the geometry of the anion and also provide qualitative descriptions of the corresponding MOs in the neutral states and structures.

As seen in Figure 3, the $16 a^{\prime}$ and $4 a^{\prime \prime}$ MOs are both delocalized over the molecular frame but have significant $\mathrm{C} 1-\mathrm{C} 2$ bonding characters. Hence, the addition of an electron to $4 a^{\prime \prime}$ shortens the bond in the anion $\left[X^{2} \mathrm{~A}^{\prime \prime}: \ldots\left(3 a^{\prime \prime}\right)^{2}\left(16 a^{\prime}\right)^{2}\left(4 a^{\prime \prime}\right)^{1}\right.$ vs. $\left.X^{1} \mathrm{~A}^{\prime}: \ldots\left(3 a^{\prime \prime}\right)^{2}\left(16 a^{\prime}\right)^{2}\right]$. This contributes to the dissimilarity of the anion and ground-state neutral structures, in addition to the internal rotation of the methyl group. Comparing $16 a^{\prime}$ and $4 a^{\prime \prime}$, the latter orbital has greater electron density along the $\mathrm{C} 1-\mathrm{C} 2$ bond, resulting in a shortening of this bond not only in the anion but also in the triplet $\left[a^{3} \mathrm{~A}^{\prime \prime}\right.$ : ... $\left.\left(3 a^{\prime \prime}\right)^{2}\left(16 a^{\prime}\right)^{1}\left(4 a^{\prime \prime}\right)^{1}\right]$, compared to the ground state $\left[X^{1} \mathrm{~A}^{\prime}\right.$ : ... $\left.\left(3 a^{\prime \prime}\right)^{2}\left(16 a^{\prime}\right)^{2}\right]$. In addition to the similar orientations of the methyl group, this effect contributes to the similarity of the triplet and anion structures. These qualitative arguments are in agreement with the optimized geometric parameters in Table I.

Given the predicted structures, one expects a broad and congested band for anion photodetachment to the singlet ground state of the neutral, attributed to the significant geometry difference with the anion, and a sharper transition for the triplet, whose geometry is similar to that of the anion. These expectations are confirmed by the broad feature A (ground-state singlet) and narrow feature B (triplet) in the anion photoelectron spectra of MG in Figure 1.

The CCSD energies (excluding the zero-point vibrational energy corrections) of the optimized anion, singlet, and triplet structures are included in Table $\mathrm{I}$, as are the $X^{1} \mathrm{~A}^{\prime}$ and $a^{3} \mathrm{~A}^{\prime \prime}$ adiabatic EAs, calculated as the differences between the corresponding neutral and anion energies $(\triangle \mathrm{E}-\mathrm{CCSD})$. Using the $\triangle \mathrm{E}$-CCSD approach, the EA of the $X^{1} \mathrm{~A}^{\prime}$ state of $\mathrm{MG}$ is predicted to be $0.759 \mathrm{eV}$, which should be compared to the origin of band $\mathrm{A}$ in the $812 \mathrm{~nm}$ MG spectrum in Figure 1(a) at $0.87(1) \mathrm{eV}$. The EA of the $a^{3} \mathrm{~A}^{\prime \prime}$ state is predicted to be $3.504 \mathrm{eV}$, compared to 3.27(2) eV determined from band $\mathrm{B}$ in Figure 1(b).

\section{B. Vertical energy gaps between the anion and neutral states}

We now compare the observed spectral bands to the vertical detachment energies predicted for the transitions to the $X^{1} \mathrm{~A}^{\prime}, a^{3} \mathrm{~A}^{\prime \prime}$, and $A^{1} \mathrm{~A}^{\prime \prime}$ neutral states of MG. As seen from the electron configurations of the four states included in the analysis (Table II), only the $X^{1} \mathrm{~A}^{\prime}$ state has a closed-shell structure; all others, including the first excited singlet $\left(A^{1} \mathrm{~A}^{\prime \prime}\right)$, are open-shell structures.

The following theoretical methods were used to determine the VDEs. First, the VDEs were obtained using the $\triangle \mathrm{E}-\mathrm{CCSD}$ methodology, i.e. by calculating the difference of the neutral and anion CCSD energies, both determined at the geometry of the anion. Although the $\triangle \mathrm{E}$-CCSD approach is commonly used, it is deficient in that these calculations do not treat the anion and the neutral on an equal footing: different reference wave functions are used in each case. Other calculations carried out in the present work employed the equation-of-motion coupled-cluster methods. Different variants of this methodology allow for accurate calculations of excitation energies by describing various pairs of electronic states (e.g., anion and neutral, ground and excited) in a consistent manner, using the same reference. ${ }^{29}$

The first EOM method used here is EOM-EA-CCSDan "electron affinity" (EA) calculation, adding an electron to the closed-shell $X^{1} \mathrm{~A}^{\prime}$ : . . . $\left(3 a^{\prime \prime}\right)^{2}\left(16 a^{\prime}\right)^{2}$ neutral reference. The second is EOM-IP-CCSD—an "ionization potential" (IP) calculation, removing either an $\alpha$ or a $\beta$ electron from the $X^{2} \mathrm{~A}^{\prime \prime}$ : $\ldots\left(3 a^{\prime \prime}\right)^{2}\left(16 a^{\prime}\right)^{2}\left(4 a^{\prime \prime}\right)^{1}$ anion reference. Unlike $\triangle \mathrm{E}$-CCSD, the EOM-EA-CCSD and EOM-IP-CCSD methods each treat the anion and the neutral using the same reference $\left(X^{1} \mathrm{~A}^{\prime}\right.$ for EA and $X^{2} \mathrm{~A}^{\prime \prime}$ for IP). The EOM-IP-CCSD calculations were extended to include the diagonal triples (dT) corrections ${ }^{30}$ to the CCSD energies, achieving the EOM-IP-CCSD(dT) level of theory. Due to the excessive computational cost of dT 
TABLE II. Properties of the anion and neutral MG states at the CCSD/aug-cc-pVTZ optimized geometry of the anion, determined using the methods indicated. All calculations used the aug-cc-pVTZ basis set. See the text for details. The computed results are compared to the corresponding experimental band positions (from Figure 1). Bold values indicate the recommended (highest-level) theory predictions.

\begin{tabular}{|c|c|c|c|c|}
\hline \multirow[b]{2}{*}{ MG state } & \multicolumn{4}{|c|}{ Relative energy (eV) } \\
\hline & Electron configuration & Vertical & Adiabatic & Method \\
\hline$X^{2} \mathrm{~A}^{\prime \prime}$ (anion) & $\ldots\left(3 a^{\prime \prime}\right)^{2}\left(16 a^{\prime}\right)^{2}\left(4 a^{\prime \prime}\right)^{1}$ & 0 & 0 & \\
\hline \multirow[t]{6}{*}{$X^{1} \mathrm{~A}^{\prime}$} & $\ldots\left(3 a^{\prime \prime}\right)^{2}\left(16 a^{\prime}\right)^{2}\left(4 a^{\prime \prime}\right)^{0}$ & 1.249 & 0.759 & $\Delta \mathrm{E}-\mathrm{CCSD}$ \\
\hline & & 1.149 & & EOM-EA-CCSD \\
\hline & & 1.271 & & EOM-IP-CCSD \\
\hline & & 1.192 & & EOM-IP-CCSD(dT) \\
\hline & & $1.249^{\mathrm{a}}$ & & EOM-IP-CCSD(dT)/SF-CCSD \\
\hline & & $1.20(4)$ & $0.87(1)$ & Experiment (band A) \\
\hline \multirow[t]{4}{*}{$a^{3} \mathrm{~A}^{\prime \prime}$} & $\ldots\left(3 a^{\prime \prime}\right)^{2}\left(16 a^{\prime}\right)^{1}\left(4 a^{\prime \prime}\right)^{1}$ & 3.566 & 3.504 & $\Delta \mathrm{E}-\mathrm{CCSD}$ \\
\hline & & 3.467 & & EOM-IP-CCSD \\
\hline & & 3.396 & & $\operatorname{EOM-IP-CCSD}(d T)$ \\
\hline & & \multicolumn{2}{|c|}{$3.27(2)^{\mathrm{b}}$} & Experiment (band B) \\
\hline \multirow[t]{4}{*}{$A^{1} \mathrm{~A}^{\prime \prime}$} & $\ldots\left(3 a^{\prime \prime}\right)^{2}\left(16 a^{\prime}\right)^{1}\left(4 a^{\prime \prime}\right)^{1}$ & 3.685 & & EOM-IP-CCSD \\
\hline & & 3.580 & & EOM-IP-CCSD(dT) \\
\hline & & $3.713^{\mathrm{c}}$ & & EOM-IP-CCSD(dT)/SF-CCSD \\
\hline & & \multicolumn{2}{|c|}{$3.614(9)^{\mathrm{b}}$} & Experiment (band C) \\
\hline
\end{tabular}

a Obtained by subtracting the $2.047 \mathrm{eV}$ singlet-triplet vertical energy gap (at the anion geometry), calculated using EOM-SF-CCSD, from the $\mathrm{VDE}=3.396 \mathrm{eV}$ corresponding to the triplet state, calculated using EOM-IP-CCSD $(\mathrm{dT})$.

${ }^{b}$ Narrow peak maxima assumed to represent both the adiabatic and vertical energy gaps between the anion and neutral states of similar equilibrium geometries.

c Obtained by adding the $0.317 \mathrm{eV}$ vertical energy gap between the $a^{3} \mathrm{~A}^{\prime \prime}$ and $A^{1} \mathrm{~A}^{\prime \prime}$ states (at the anion geometry), calculated using EOM-SF-CCSD, to the VDE $=3.396 \mathrm{eV}$ corresponding to the $a^{3} \mathrm{~A}^{\prime \prime}$ state, calculated using EOM-IP-CCSD $(\mathrm{dT})$.

corrections, they were not attempted for other types of EOM calculations described in this work.

Finally, we employed an aggregate method. The vertical gaps between the $X^{1} \mathrm{~A}^{\prime}, a^{3} \mathrm{~A}^{\prime \prime}$, and $A^{1} \mathrm{~A}^{\prime \prime}$ neutral states were calculated using the spin-flip (SF) methodology, EOM-SF-CCSD, using the high-spin $\left(M_{S}=1\right)^{3}$ $\mathrm{A}^{\prime \prime}: \ldots\left(3 a^{\prime \prime}\right)^{2}\left(16 a^{\prime}\right)^{1}\left(4 a^{\prime \prime}\right)^{1}$ reference. These energy gaps were then combined with the EOM-IP-CCSD(dT) VDE corresponding to the triplet state, to yield the aggregate

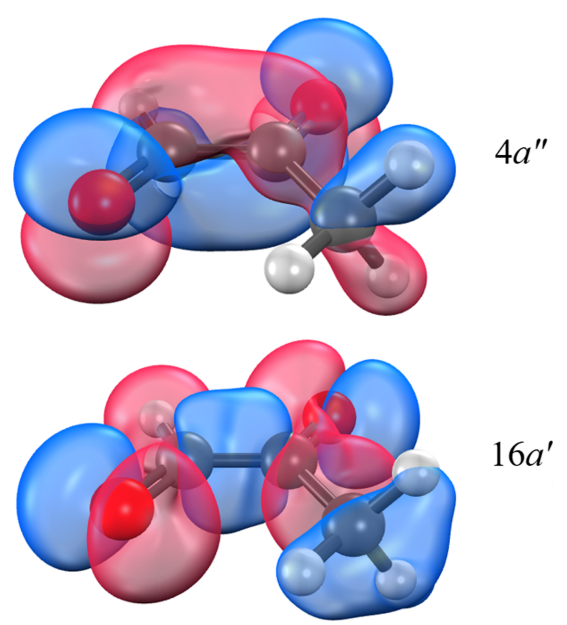

FIG. 3. The $16 a^{\prime}-\alpha$ (HOMO-1) and the $4 a^{\prime \prime}-\alpha$ HOMO of the MG anion from CCSD/aug-cc-pVTZ calculations.
EOM-IP-CCSD(dT)/SF-CCSD predictions for the VDEs to the two singlets.

All of the above EOM studies were single-point calculations using the aug-cc-pVTZ basis set and the CCSD/augcc-pVTZ optimized geometry of the anion described in Figure 2 and Table I. The results are summarized in Table II, alongside the adiabatic EA values determined using the $\Delta \mathrm{E}$ CCSD method. The highest-level VDE predictions, i.e., EOMIP-CCSD(dT)/aug-cc-pVTZ, are boldfaced in Table II and indicated by the green vertical bars overlaying the experimental spectra in Figures 1(a) and 1(b). The experimental VDE and EA values are also included in Table II for comparison.

The VDE values predicted by the different theoretical methods for the A spectral band $\left(X^{1} \mathrm{~A}^{\prime}\right.$ state of $\left.\mathrm{MG}\right)$ all fall within or close to the uncertainty range of the experimental result $\mathrm{VDE}=1.20(4) \mathrm{eV}$. The highest-level prediction, $1.192 \mathrm{eV}$, obtained by the EOM-IP-CCSD(dT)/aug-cc-pVTZ method, is in perfect agreement with the experiment. For transition from the anion to the triplet, the EOM-IPCCSD(dT)/aug-cc-pVTZ method predicts a VDE of $3.396 \mathrm{eV}$, compared to the $3.27(2) \mathrm{eV}$ experimental value. The predicted VDE coincides with the second major peak of the $\mathrm{B}$ band, rather than the most intense origin peak, as seen in Figure 1(b). The slight discrepancy may be the result of minor errors in the equilibrium molecular geometries, which were optimized at the CCSD level without the triples corrections. 
For detachment to the open-shell singlet, the $A^{1} \mathrm{~A}^{\prime \prime}$ state, the VDE of $3.580 \mathrm{eV}$ predicted by the EOM-IP-CCSD(dT) method, is very close to the observed band $\mathrm{C}$ position in Figure 1(b), VDE $=3.614(9) \mathrm{eV}$. For all three photodetachment bands (A, B, and $\mathrm{C}$ ) and the corresponding neutral states $\left(X^{1} \mathrm{~A}^{\prime}, a^{3} \mathrm{~A}^{\prime \prime}\right.$, and $\left.A^{1} \mathrm{~A}^{\prime \prime}\right)$, inclusion of the diagonal-triples corrections in the EOM-IP-CCSD calculations significantly improves the agreement with the experiment.

Although the minimum-energy geometry of the $A^{1} \mathrm{~A}^{\prime \prime}$ state was not determined, its nominal electron configuration is the same as for the triplet $\left(a^{3} \mathrm{~A}^{\prime \prime}\right)$, as seen in Table II. Therefore, we expect the $A^{1} \mathrm{~A}^{\prime \prime}$ and $a^{3} \mathrm{~A}^{\prime \prime}$ equilibrium structures to be qualitatively similar to each other, and hence both be similar to that of the anion. The sharp appearance of the $\mathrm{C}$ band in Figure 1(b) is consistent with this expectation. Moreover, the observed relative intensities of the $\mathrm{B}$ and $\mathrm{C}$ bands qualitatively reflect the respective multiplicities of the triplet and singlet states (although the intensity of the $\mathrm{C}$ band may also be suppressed by the Wigner effect ${ }^{34}$ in the proximity of the spectral cutoff).

\section{Franck-Condon simulation of the triplet band}

To confirm the assignment of the higher-energy transitions, we performed a Franck-Condon (FC) simulation of the triplet band $\left(a^{3} \mathrm{~A}^{\prime \prime} \leftarrow X^{2} \mathrm{~A}^{\prime \prime}\right)$. The primary goal was to verify that the sharp peak labeled $C$ in Figure 1(b) was separate from the $\mathrm{B}$ band and belongs to a different electronic state of MG. The FC procedure was identical to that used previously, ${ }^{1}$ with one notable exception: none of the anion-toneutral displacement vectors proved necessary in the present work.

Briefly, the FC simulation was performed using the PESCAL program ${ }^{32,33}$ and the Gaussian-optimized CCSD/aug-cc-pVTZ geometries of the anion and the triplet neutral. The PESCAL-calculated FC intensities were multiplied by an $\mathrm{eKE}^{3 / 2}$ pre-factor, accounting for the Wigner-like ${ }^{34}$ scaling of the electronic part of the photodetachment cross section near zero eKE. ${ }^{35}$ The procedure yielded the stick spectrum shown in Figure 4. The 0-0 transition energy was set to $3.27 \mathrm{eV}$, to match the first (most intense) peak of band B in Figure 1(b).

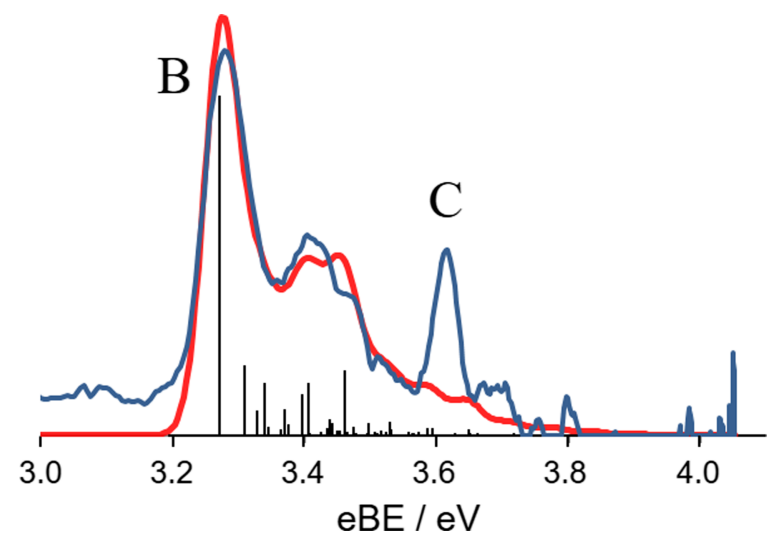

FIG. 4. The blue line is a magnified portion of the $306 \mathrm{~nm}$ anion photoelectron spectrum of methylglyoxal reproduced from Figure 1(b). The results of the Franck-Condon simulation for the triplet transitions are shown in black as discrete transitions, while the red spectrum is the simulation convoluted with a Gaussian function representing instrument resolution. See the text for details.
Aside from this energy-scale calibration, no adjustments of the $a b$ initio anion geometry (in order to match the simulation to the experimental spectrum) were necessary. The result in Figure 4 represents the raw, unadjusted FC simulation of the spectrum.

To obtain a smooth simulation, the scaled stick spectrum represented in the speed domain was convoluted with an instrumental resolution function, taken to be a Gaussian of a FWHM $=2 \times 10^{4} \mathrm{~m} / \mathrm{s}$. The width of the instrumental function was determined from $\mathrm{O}^{-}$photodetachment under similar experimental conditions. The convoluted speed spectrum was then transformed into the eBE domain using the appropriate Jacobian, and the result is shown in Figure 4 (red), overlaid with the corresponding portion of the experimental photoelectron spectrum (blue).

The FC spectrum accounts for the two major features of band $\mathrm{B}$ visible in the experimental spectrum and indicates that the first, most intense peak is indeed the 0-0 transition of the $a^{3} \mathrm{~A}^{\prime \prime} \leftarrow X^{2} \mathrm{~A}^{\prime \prime}$ band. It also confirms that the peak labeled $\mathrm{C}$ in Figure 1(b) belongs to a different electronic transition. Its assignment as the $A^{1} \mathrm{~A}^{\prime \prime} \leftarrow X^{2} \mathrm{~A}^{\prime \prime}$ band is, therefore, further justified, consistent with the theory predictions described in Sec. IV B.

\section{SUMMARY}

Using anion photoelectron imaging spectroscopy, we observed and characterized the anion of methylglyoxal and three lowest electronic states of neutral MG: the closed-shell singlet ground state $\left(X^{1} \mathrm{~A}^{\prime}\right)$, the lowest triplet state $\left(a^{3} \mathrm{~A}^{\prime \prime}\right)$, and the open-shell singlet state $\left(A^{1} \mathrm{~A}^{\prime \prime}\right)$. From ab initio geometry optimizations, the equilibrium geometry of the anion $\left(X^{2} \mathrm{~A}^{\prime \prime}\right)$ and the triplet was found to be similar to each other, while that of the ground-state singlet qualitatively differs from both the anion and the triplet, mainly by a $60^{\circ}$ internal rotation of the methyl group with respect to the molecular frame. Accordingly, the $X^{1} \mathrm{~A}^{\prime} \leftarrow X^{2} \mathrm{~A}^{\prime \prime}$ photodetachment transition appears as a broad, congested band, while the triplet band presents as a partially resolved, rather narrow progression, with the most intense peak corresponding to the origin. The geometry of the $A^{1} \mathrm{~A}^{\prime \prime}$ state was not optimized, but based on its nominal electron configuration, we expect the open-shell singlet equilibrium to have a similar structure to that of the triplet, and hence the anion. This expectation is confirmed by the $A^{1} \mathrm{~A}^{\prime \prime} \leftarrow X^{2} \mathrm{~A}^{\prime \prime}$ photodetachment transition, which is dominated by a single sharp peak assigned to the transition origin.

From the experimental spectra, the adiabatic electron affinities of methylglyoxal in the ground and excited states were determined for the first time. The electron affinity of the ground state is $\operatorname{EA}\left(X^{1} \mathrm{~A}^{\prime}\right)=0.87(1) \mathrm{eV}$, compared to $1.10(2) \mathrm{eV}$ for the unsubstituted glyoxal molecule. ${ }^{1}$ The EAs of the excited states of $\mathrm{MG}$ were also determined: $\operatorname{EA}\left(a^{3} \mathrm{~A}^{\prime \prime}\right)$ $=3.27(2) \mathrm{eV}$ and $\operatorname{EA}\left(A^{1} \mathrm{~A}^{\prime \prime}\right)=3.614(9) \mathrm{eV}$. Consistent with the past phosphorescence ${ }^{15}$ and fluorescence ${ }^{16}$ measurements, the results correspond to a $2.40(2) \mathrm{eV}$ adiabatic energy gap between the ground-state singlet and the triplet and a $2.74(1)$ $\mathrm{eV}$ gap between the closed-shell $\left(X^{1} \mathrm{~A}^{\prime}\right)$ and open-shell $\left(A^{1} \mathrm{~A}^{\prime \prime}\right)$ singlets. 
While the anion photodetachment to the $a^{3} \mathrm{~A}^{\prime \prime}$ and $A^{1} \mathrm{~A}^{\prime \prime}$ neutral states produces the neutral species near the respective equilibria, the lowest-energy photodetachment transition involves significant geometry relaxation in the $X^{1} \mathrm{~A}^{\prime}$ state. The corresponding vertical detachment energy is determined experimentally as $\mathrm{VDE}=1.20(4) \mathrm{eV}$, compared to the $0.87(1)$ $\mathrm{eV}$ adiabatic EA of the $X^{1} \mathrm{~A}^{\prime}$ state, yielding a $0.33(4) \mathrm{eV}$ relaxation energy. The above VDE of the $X^{1} \mathrm{~A}^{\prime} \leftarrow X^{2} \mathrm{~A}^{\prime \prime}$ band is in excellent agreement with the EOM-IP-CCSD(dT)/augcc-pVTZ prediction of $1.192 \mathrm{eV}$. Similar agreements are demonstrated for the excited-state transitions.

\section{ACKNOWLEDGMENTS}

We acknowledge the support of this work by the U.S. National Science Foundation (Grant No. CHE-1266152).

${ }^{1}$ T. Xue, A. R. Dixon, and A. Sanov, Chem. Phys. Lett. 660, 205 (2016).

${ }^{2}$ J. P. DiGangi et al., Atmos. Chem. Phys. 12, 9529 (2012).

${ }^{3}$ K. F. Ho, S. S. H. Ho, W. T. Dai, J. J. Cao, R. J. Huang, L. W. Tian, and W. J. Deng, Environ. Monit. Assess. 186, 2835 (2014).

${ }^{4}$ C. J. Kampf, A. L. Corrigan, A. M. Johnson, W. Song, P. Keronen, R. Konigstedt, J. Williams, L. M. Russell, T. Petaja, H. Fischer, and T. Hoffmann, Atmos. Chem. Phys. 12, 6145 (2012).

${ }^{5}$ M. Vrekoussis, F. Wittrock, A. Richter, and J. P. Burrows, Atmos. Chem. Phys. 9, 4485 (2009).

${ }^{6}$ H. E. Krizner, D. O. De Haan, and J. Kua, J. Phys. Chem. A 113, 6994 (2009).

${ }^{7}$ D. O. De Haan, A. L. Corrigan, M. A. Tolbert, J. L. Jimenez, S. E. Wood, and J. J. Turley, Environ. Sci. Technol. 43, 8184 (2009).

${ }^{8}$ R. Zhao, A. K. Y. Lee, and J. P. D. Abbatt, J. Phys. Chem. A 116, 6253 (2012).

${ }^{9}$ B. M. Connelly, D. O. De Haan, and M. A. Tolbert, J. Phys. Chem. A 116, 6180 (2012).

${ }^{10}$ A. G. Carlton, B. J. Turpin, K. E. Altieri, S. Seitzinger, A. Reff, H. J. Lim, and B. Ervens, Atmos. Environ. 41, 7588 (2007).

${ }^{11}$ M. M. Galloway, M. H. Powelson, N. Sedehi, S. E. Wood, K. D. Millage, J. A. Kononenko, A. D. Rynaski, and D. O. De Haan, Environ. Sci. Technol. 48, 14417 (2014).
${ }^{12}$ R. N. Compton, P. W. Reinhardt, and H. C. Schweinler, Int. J. Mass Spectrom. Ion Phys. 49, 113 (1983).

${ }^{13}$ J. E. Bartmess, "Negative ion energetics data," in NIST Chemistry WebBook, NIST Standard Reference Database Number 69, edited by P. J. Linstrom and W. G. Mallard (National Institute of Standards and Technology, Gaithersburg, MD, 20899, 2017), http://webbook.nist.gov.

${ }^{14}$ D. C. Rawlings and E. R. Davidson, J. Chem. Phys. 72, 6808 (1980).

${ }^{15}$ L. H. Spangler and D. W. Pratt, J. Chem. Phys. 84, 4789 (1986).

${ }^{16}$ M. Gurnick, J. Chaiken, T. Benson, and J. D. McDonald, J. Chem. Phys. 74, 99 (1981).

${ }^{17}$ L. Velarde, T. Habteyes, and A. Sanov, J. Chem. Phys. 125, 114303 (2006).

${ }^{18}$ A. Sanov and R. Mabbs, Int. Rev. Phys. Chem. 27, 53 (2008).

${ }^{19}$ A. R. Dixon, T. Xue, and A. Sanov, Angew. Chem., Int. Ed. 54, 8764 (2015).

${ }^{20}$ A. R. Dixon, T. Xue, and A. Sanov, J. Chem. Phys. 144, 234305 (2016).

${ }^{21}$ M. A. Johnson and W. C. Lineberger, "Pulsed methods for cluster ion spectroscopy," in Techniques for the Study of Ion Molecule Reactions, edited by J. M. Farrar and W. H. Saunders (Wiley, New York, 1988), pp. 591-635.

${ }^{22}$ W. C. Wiley and I. H. McLaren, Rev. Sci. Instrum. 26, 1150 (1955).

${ }^{23}$ A. T. J. B. Eppink and D. H. Parker, Rev. Sci. Instrum. 68, 3477 (1997).

${ }^{24}$ A. J. R. Heck and D. W. Chandler, Annu. Rev. Phys. Chem. 46, 335 (1995).

${ }^{25}$ V. Dribinski, A. Ossadtchi, V. A. Mandelshtam, and H. Reisler, Rev. Sci. Instrum. 73, 2634 (2002).

${ }^{26}$ D. M. Neumark, K. R. Lykke, T. Andersen, and W. C. Lineberger, Phys. Rev. A 32, 1890 (1985).

${ }^{27}$ S. J. Cavanagh, S. T. Gibson, M. N. Gale, C. J. Dedman, E. H. Roberts, and B. R. Lewis, Phys. Rev. A 76, 052708 (2007).

${ }^{28}$ Y. Shao et al., Phys. Chem. Chem. Phys. 8, 3172 (2006).

${ }^{29}$ A. I. Krylov, Annu. Rev. Phys. Chem. 59, 433 (2008).

${ }^{30}$ P. U. Manohar and A. I. Krylov, J. Chem. Phys. 129, 194105 (2008).

${ }^{31}$ M. J. Frisch et al., Gaussian 09, Revision A.02, Gaussian, Inc., Wallingford, CT, 2009.

${ }^{32}$ K. M. Ervin, T. M. Ramond, G. E. Davico, R. L. Schwartz, S. M. Casey, and W. C. Lineberger, J. Phys. Chem. A 105, 10822 (2001).

${ }^{33} \mathrm{P}$. Chen, in Unimolecular and Bimolecular Reactions Dynamics, edited by C.-Y. Ng, T. Baer, and I. Powis (John Wiley \& Sons, Chichester, 1994), pp. 371.

${ }^{34}$ E. P. Wigner, Phys. Rev. 73, 1002 (1948).

${ }^{35}$ D. Khuseynov, A. R. Dixon, D. J. Goebbert, and A. Sanov, J. Phys. Chem. A 117, 10681 (2013). 